\title{
Hábitos alimentarios del tiburón martillo Sphyrna lewini (Griffith \& Smith, 1834) (Chondrichthyes) en el Pacífico ecuatoriano
}

\author{
Feeding habits of the scalloped hammerhead shark Sphyrna lewini (Griffith \& Smith, 1834) \\ (Chondrichthyes) in the Ecuadorian Pacific \\ Colombo Estupiñán-Montaño ${ }^{1 *}$, Luis G. Cedeño-Figueroa ${ }^{1}$ y Felipe Galván-Magaña² \\ ${ }^{1}$ Universidad Laica 'Eloy Alfaro’ de Manabí (ULEAM). Facultad Ciencias del Mar. P.O. Box 27-32. Manta, Manabí, Ecuador \\ ${ }^{2}$ Centro Interdisciplinario de Ciencias Marinas. Apartado Postal 592, C.P. 23000. La Paz, Baja California Sur, México \\ *goliathcem@gmail.com
}

\begin{abstract}
This study analyzed the diet of 116 specimens of scalloped hammerhead, Sphyrna lewini caught by the shark fleet in the Ecuadorian Pacific. The sampling was between January and December 2004. The index of relative importance (\%) showed the highest values for the cephalopods Histioteuthis spp. (22.7\%) and Dosidicus gigas (21.9\%). In relation to feeding by maturity stage and sex, the juvenile females predated mainly on D. gigas (58.5\%); whereas the adult females feed mainly on Octopus spp. (47.6\%), Histioteuthis spp. (18.7\%) and Sthenoteuthis oualaniensis (16.3\%). The juvenile males fed on cephalopods Mastigoteuthis spp. (12.1), Histioteuthis spp. (10.2) and Ancistrocheirus lesueuri (7.9), while the adult males predated on D. gigas (53.7\%) and Histioteuthis spp. (14.9\%). Considering the prey habitat, S. lewini is an oceanic predator which consumed oceanic prey, mainly cephalopods; whereas the juvenile sharks can predate on oceanic cephalopods and also on coastal prey from benthic habitat such as fish and crustaceans.
\end{abstract}

Key words: Diet, Sphyrnidae
Resumen.- Este estudio analizó la dieta de 116 ejemplares del tiburón martillo Sphyrna lewini capturados por la flota tiburonera que pesca en el Pacífico ecuatoriano. Las muestras se recolectaron de enero a diciembre de 2004. De acuerdo al índice de importancia relativa, los cefalópodos Histioteuthis spp. (22,7\%) y Dosidicus gigas (21,9\%) fueron las presas principales. Con respecto al sexo y estado de madurez, las hembras juveniles consumieron principalmente $D$. gigas (58,5\%); mientras que las adultas consumieron principalmente Octopus spp. (47,6\%), Histioteuthis spp. (18,7\%) у Sthenoteuthis oualaniensis (16,3\%). Los machos juveniles se alimentaron de los cefalópodos Mastigoteuthis spp. (12,1), Histioteuthis spp. $(10,2)$ y Ancistrocheirus lesueuri $(7,9)$, mientras que los adultos depredaron sobre $D$. gigas $(53,7 \%)$ e Histioteuthis spp. (14,9\%). Con base en la información de los hábitats de las presas, se considera que S. lewini es un depredador que consume principalmente presas de hábitat oceánico principalmente cefalópodos; mientras que los tiburones juveniles junto con consumir cefalópodos de zonas oceánicas, se alimentan de presas de hábitats costeros y bentónicos como peces y crustáceos.

Palabras clave: Dieta, Sphyrnidae

\section{Introducción}

La falta de información y conocimientos biológico sobre tiburones en el Ecuador y en el mundo no permite establecer planes de manejo o conservación adecuados para la protección de las especies de tiburones más explotadas comercialmente. Por ello es importante realizar investigaciones que favorezcan el incremento de los conocimientos de las especies de tiburones en cada país, los cuales deben incluir el estudio de su biología básica tales como reproducción, edad, crecimiento, hábitos alimenticios y comportamiento.

El estudio de los hábitos alimenticios permite comprender el papel funcional de los organismos dentro de las comunidades marinas y aporta información de zonas importantes de alimentación y crianza de tiburones para poder recomendar, junto con otros estudios biológicos, medidas de conservación o manejo adecuado de especies de tiburones (Galván et al. 1989).

Los tiburones actúan como carroñeros ayudando a eliminar de las aguas los animales muertos y enfermos, manteniendo el equilibrio ecológico trófico de los ecosistemas marinos. A pesar de la importancia ecológica que los tiburones desempeñan en su ambiente, solo se cuenta con información biológica de algunas especies de tiburones a nivel mundial. En este contexto, el presente 
estudio está enfocado en el tiburón martillo, Sphyrna lewini (Griffith \& Smith, 1834), el cual se considera como especie vulnerable según la Unión de la conservación de la Naturaleza (IUCN), debido a la disminución de sus poblaciones a nivel mundial.

Esta especie habita aguas costeras y oceánicas de todos los mares tropicales del mundo (Compagno 1984, Robertson \& Allen 2002). En el Océano Pacífico oriental, se distribuye desde Baja California Sur, México hasta el norte de Perú (Compagno 1984, Robertson \& Allen 2002).

Los individuos juveniles del tiburón martillo habitan principalmente áreas costeras, llegando a formar agrupaciones con predominancia de hembras; mientras que los adultos se les encuentra viviendo solitarios o en grupos menores (Klimley 1981, Klimley et al. 1981). Los estudios realizados en el Golfo de California sobre hábitos alimenticios de esta especie de tiburón, indican que los juveniles se alimentan principalmente de peces bentónicos y neríticos; mientras que los adultos consumen peces neríticos y epipelágicos, así como cefalópodos, rayas, langostas, camarones y cangrejos (Klimley 1982, Galván et al. 1989, Aguilar-Castro 2003, Torres-Rojas et al. 2006).

Smale \& Cliff (1998) describieron la presencia de cefalópodos en la dieta de cuatro especies de tiburones Galeocerdo cuvier, Sphyrna lewini, S. zygaena y S. mokarran de Kwazulu-Natal, Sudáfrica; mientras que en Ecuador se reporta el trabajo realizado por Castañeda \& Sandoval (2007), acerca de los hábitos alimenticios de S. lewini. Por lo tanto, este estudio, tiene como objetivo describir el espectro dietario de S. lewini en aguas ecuatorianas y además determinar las posibles diferencias de hábitos tróficos entre machos y hembras y su estadio de madurez.

\section{Material y métodos}

Se recolectaron estómagos de 116 tiburones martillo provenientes de aguas ecuatorianas durante enero a diciembre de 2004. El área de estudio estuvo comprendida entre $02^{\circ} \mathrm{N}$ y $02^{\circ} \mathrm{S}$ y desde la costa hasta $84^{\circ} \mathrm{W}$. El puerto de desembarque de tiburones fue Manta, en Ecuador. De cada ejemplar se registró su longitud total (LT). Posteriormente se extrajo el tracto digestivo de cada ejemplar mediante la disección de los organismos. Los contenidos estomacales fueron tamizados a través de una red de $1,5 \mathrm{~mm}$ de luz de malla, y posteriormente depositados en bolsas de plástico y preservados en hielo para su traslado al laboratorio. En el momento de la disección, se determinó el estado de madurez sexual de los tiburones, el que fue clasificado como juveniles y adultos, basado en observaciones directas del aparato reproductor y según la escala de madurez propuesta por Bejarano (2007).

El contenido estomacal fue separado y las presas fueron clasificadas según su grupo taxonómico, identificándose hasta el menor taxón posible, dependiendo del grado de digestión que éstas presentaban. Para la determinación taxonómica se utilizaron diferentes claves de identificación, en el caso de los peces se utilizaron las claves de Clothier (1950), Rubio (1988), Fischer et al. (1995), Chirichigno (1998), García-Godos (2001); mientras que para identificar cefalópodos, se usaron las claves de Wolff (1984) y Clarke (1986). Debido al estado de digestión avanzado de los cefalópodos, la identificación se basó en las estructuras del aparato mandibular. La identificación de los crustáceos se realizó mediante su exoesqueleto, utilizando las claves de Fischer et al. (1995).

Para determinar si el número de estómagos era suficiente para describir la dieta de $S$. lewini, se aplicó una curva acumulativa de especies presa basada en la diversidad de presas identificadas. Para la curva acumulativa se utilizaron los programas Estimate 7.0 (Colwell 2000) y Statistica 7.0 (StatSoft 2001).

El análisis cuantitativo del contenido estomacal se realizó utilizando los métodos numérico $(\mathrm{N})$, gravimétrico $(\mathrm{P})$ y de frecuencia de ocurrencia (FO) (Hyslop 1980). Asímismo, se empleó el índice de importancia relativa (IIR) propuesto por Pinkas et al. (1971), el cual incorpora los tres métodos anteriores mediante la siguiente fórmula: IIR $=(\% \mathrm{~N}+\% \mathrm{P})$ * \%FO. Posteriormente esta fórmula fue transformada siguiendo a Cortés (1997), para obtener los valores en porcentajes y facilitar las comparaciones, $\%$ IIRi $=100 *$ IIRi / $\Sigma$ IIR.

De igual manera, se determinó la amplitud del nicho trófico mediante el índice estandarizado de Levin (Krebs 1989), representado de la siguiente manera:

$$
B i=1 / n-1\left\{\left(1 / \sum P i j^{2}\right)-1\right\}
$$

donde, $n$ es el número de presas y Pij es la proporción de la dieta del depredador $i$ sobre la presa $j$. Este índice presenta un intervalo de valores que va de 0 a 1 ; valores menores a 0,6 indican que el depredador utiliza un bajo número de recursos y presenta una preferencia por ciertas presas; por lo tanto, sería un depredador especialista; valores mayores a 0,6 indican dietas de depredadores generalistas, los cuales utilizan los recursos sin ninguna selección (Labropoulou \& Eleftheriou 1997).

Asimismo se realizó un análisis de traslapamiento trófico con el fin de determinar probables diferencias en 
la dieta entre sexo y estado de madurez sexual por medio del índice de Morisita-Horn (Smith \& Zaret 1982):

$$
C \lambda=2 \frac{\sum_{i=1}^{n}\left(P_{x i} * P_{y i}\right)}{\left(\sum_{i=1}^{n} P_{x i}{ }^{2}+\sum_{i=1}^{n} P_{y i}{ }^{2}\right)}
$$

donde: $\mathrm{C} \lambda$ = índice de Morisita-Horn entre sexos o estado de madurez sexual $x$ y entre sexo o madurez sexual $y, P x i$ = proporción de la presa $i$ del total de pesas consumidas por el sexo o estado de madurez $x$, Pyi = proporción de la presa $i$ del total de presas consumidas por el sexo o estado de madurez sexual $y, n=$ número total de presas. Este índice varía entre 0 y 1 , y se considera como un traslape biológico significativo cuando el valor excede de 0,6 (Langton 1982).

\section{Resultados}

De los 116 estómagos analizados, 102 (88\%) tenían contenido estomacal. De acuerdo al resultado de la curva acumulativa (Fig. 1), se observó que a partir de 80 estómagos se puede encontrar al menos el $90 \%$ de las presas que componen el espectro dietario total de Sphyrna lewini. Se identificaron 34 componentes alimentarios, sin considerar aquellos restos organismos indeterminados, donde 15 fueron moluscos (principalmente cefalópodos), 18 peces y un crustáceo (Tabla 1). Las presas con mayor porcentaje numérico fueron los cefalópodos (51,2\%) y los peces $(45,1 \%)$. Gravimétricamente, las presas de mayor importancia fueron los cefalópodos Dosidicus gigas, Histioteuthis spp. y Onychoteuthis banksii (Tabla 1). Los componentes alimenticios más frecuentes en los estómagos fueron los cefalópodos Histioteuthis spp., Mastigoteuthis spp., Anchoa spp., D. gigas (13,7\%) y Octopus spp. (12,75\%), y los peces teleósteos Merluccius gayi (12,75\%) y Auxis thazard (10,8\%) (Tabla 1). De acuerdo con al IIR, los cefalópodos Histioteuthis spp. (22,7\%) y D. gigas (21,9\%) fueron los más importantes.

\section{Análisis trófico por sexo}

De los 116 tiburones analizados, 46 fueron machos y 70 hembras. En ambos sexos se identificaron tres grupos de presas: cefalópodos, peces y crustáceos, siendo los cefalópodos las presas principales en ambos sexos (Tabla 2a). Al menos 17 presas fueron compartidas entre machos y hembras (Tabla 2a).

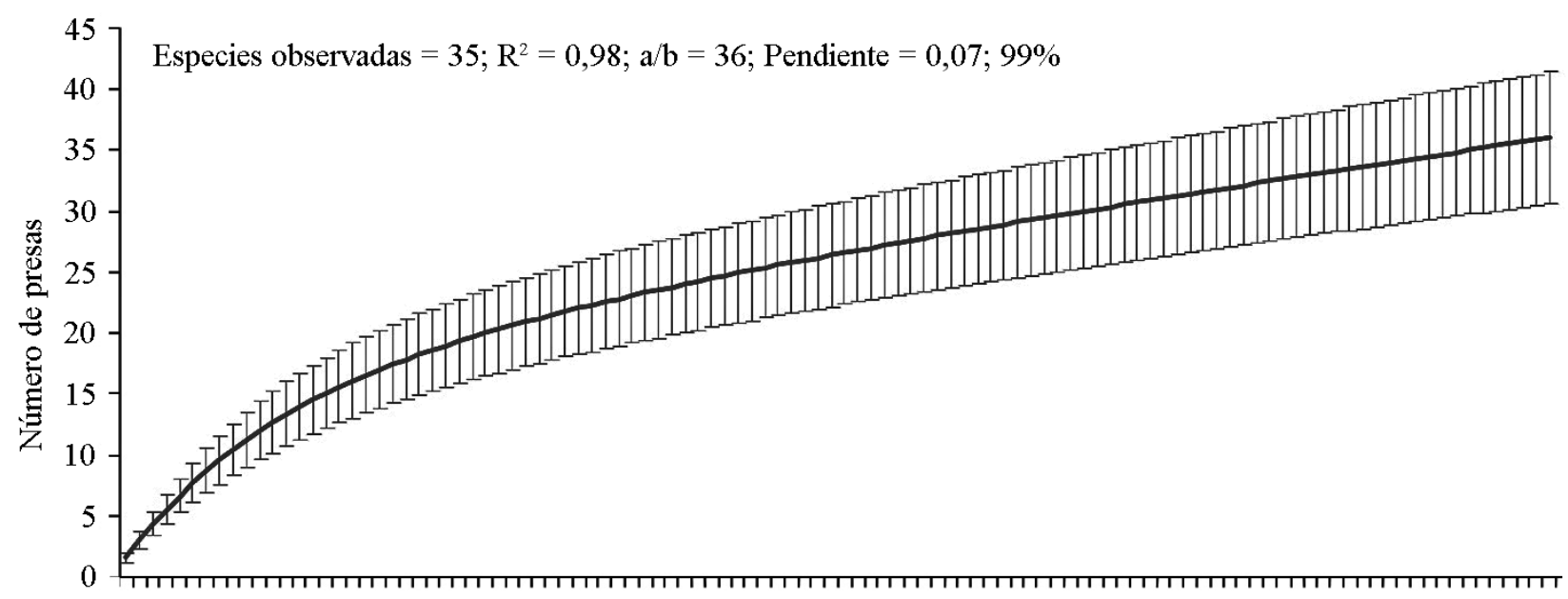

147101316192225283134374043464952555861646770737679828588919497100103106

Número de estómagos

Figura 1

Curva acumulativa de presas de $S$. lewini

Prey accumulation curves in S. lewini 


\section{Tabla 1}

Espectro trófico de 116 ejemplares del tiburón martillo $S$. lewini en Ecuador, expresado en valores porcentuales de los métodos numérico $(\mathrm{N})$, gravimétrico $(\mathrm{P})$, frecuencia de ocurrencia (FO) e índice de importancia relativa (IIR)

Trophic spectrum from 116 individuals of scalloped hammerhead S. lewini in Ecuador, expressed in percent values of numerical $(\mathrm{N})$, gravimetrical $(\mathrm{P})$ frequency of occurrence $(\mathrm{FO})$ and index of relative importance (IIR)

\begin{tabular}{|c|c|c|c|c|}
\hline Especies presa & $\% \mathrm{~N}$ & $\% \mathrm{P}$ & $\% \mathrm{FO}$ & $\%$ IIR \\
\hline \multicolumn{5}{|l|}{ Cephalopoda } \\
\hline Lolliguncula (Loliolopsis) diomedeae & 9,41 & 2,76 & 6,86 & 3,03 \\
\hline Ancistrocheirus lesueuri & 1,73 & 3,87 & 5,88 & 1,20 \\
\hline Gonatus spp. & 0,74 & 0,35 & 2,94 & 0,12 \\
\hline Histioteuthis spp. & 10,4 & 21,51 & 19,61 & 22,72 \\
\hline Mastigoteuthis spp. & 5,45 & 1,69 & 14,71 & 3,81 \\
\hline Octopoteuthis spp. & 1,98 & 3,75 & 5,88 & 1,22 \\
\hline Dosidicus gigas & 7,43 & 36,59 & 13,73 & 21,94 \\
\hline Ommastrephes bartramii & 0,74 & $<0,01$ & 1,96 & 0,05 \\
\hline Sthenoteuthis oualaniensis & 2,72 & 6,24 & 5,88 & 1,91 \\
\hline Onychoteuthis banksii & 3,22 & 12,77 & 2,94 & 1,71 \\
\hline Thysanoteuthis rhombus & 0,25 & 0,01 & 0,98 & 0,01 \\
\hline Argonauta spp. & 0,25 & $<0,01$ & 0,98 & 0,01 \\
\hline Octopus spp. & 4,46 & $<0,01$ & 12,75 & 2,06 \\
\hline Pholidoteuthis boschmaii & 0,25 & $<0,01$ & 0,98 & 0,01 \\
\hline Restos de cefalópodos & 1,98 & 2,14 & 6,86 & 1,03 \\
\hline Gastropoda indeterminado & 0,25 & $<0,01$ & 0,98 & 0,01 \\
\hline Sub-total & 51,26 & 91,68 & & 60,84 \\
\hline \multicolumn{5}{|l|}{ Osteichthyes } \\
\hline Muraena spp. & 0,25 & 0,04 & 0,98 & 0,01 \\
\hline Ophichthus spp. & 1,49 & 0,58 & 4,90 & 0,37 \\
\hline Synodus spp. & 0,25 & 0,27 & 0,98 & 0,02 \\
\hline Cypselurus callopterus & 0,25 & 0,03 & 0,98 & 0,01 \\
\hline Oxyporhamphus micropterus & 0,25 & 0,02 & 0,98 & 0,01 \\
\hline Anchoa spp. & 7,93 & 0,67 & 15,69 & 4,33 \\
\hline Merluccius gayi & 13,86 & 0,24 & 12,75 & 6,53 \\
\hline Benthosema panamense & 2,97 & $<0,01$ & 5,88 & 0,63 \\
\hline Mugil cephalus & 0,25 & 0,46 & 0,98 & 0,03 \\
\hline Chloroscombrus orqueta & 0,25 & 0,01 & 0,98 & 0,01 \\
\hline Larimus argenteus & 1,73 & 0,04 & 4,90 & 0,32 \\
\hline Hemanthias peruanus & 0,25 & 0,19 & 0,98 & 0,02 \\
\hline Auxis thazard & 2,97 & 1,93 & 10,78 & 1,92 \\
\hline Sarda orientalis & 0,25 & 0,36 & 0,98 & 0,02 \\
\hline Restos de Serranidae & 0,25 & 0,34 & 0,98 & 0,02 \\
\hline Restos de Scombridae & 0,25 & 0,06 & 0,98 & 0,01 \\
\hline Restos de Paralichthyidae & 0,25 & 0,01 & 0,98 & 0,01 \\
\hline Restos de peces no identificados & 11,39 & 2,65 & 45,10 & 22,98 \\
\hline Sub-total & 45,09 & 7,90 & & 37,25 \\
\hline \multicolumn{5}{|l|}{ Crustacea } \\
\hline Penaeus spp. & 3,71 & 0,43 & 12,75 & 1,92 \\
\hline Sub-total & 3,71 & 0,43 & & 1,92 \\
\hline
\end{tabular}


Tabla 2

Espectro trófico por sexo (a) y estadio de madurez (b) de S. lewini en Ecuador, expresado con el índice de importancia relativa (\% IIR)

Trophic spectrum by sex (a) and maturity stage (b) of S. lewini in Ecuador, expressed in percentage of index of relative importance (\%IIR)

\begin{tabular}{|c|c|c|c|c|c|c|c|}
\hline \multirow[b]{2}{*}{ Especies } & \multicolumn{2}{|c|}{$\%$ IIR } & \multirow[b]{2}{*}{ Especies } & \multicolumn{2}{|c|}{ \%IIR Machos } & \multicolumn{2}{|c|}{ \%IIR Hembras } \\
\hline & Machos & Hembras & & Juveniles & Adultos & Juveniles & Adultos \\
\hline & & & & $\mathrm{N}=26$ & $\mathrm{~N}=20$ & $\mathrm{~N}=54$ & $\mathrm{~N}=16$ \\
\hline Mollusca & & & Mollusca & & & & \\
\hline Ancistrocheirus lesueuri & 2,05 & 0,79 & Ancistrocheirus lesueuri & 7,79 & & 0,38 & 3,56 \\
\hline Dosidicus gigas & 17,19 & 18,34 & Argonauta spp. & & & 0,02 & \\
\hline Histioteuthis spn. & 16.43 & 28,11 & Dosidicus gigas & & 53,66 & 58,52 & \\
\hline Histloteuthis spp. & 16,43 & 28,11 & Gasterópodo no identificado & & & 0,02 & \\
\hline Lolliguncula (Loliolopsis) diomedeae & 5,84 & 0,74 & Gonatus spp. & 4,21 & & 0,11 & \\
\hline Mastigoteuthis spp. & 2,87 & 4,70 & Histioteuthis spp. & 10,19 & 14,86 & 10,52 & 18,71 \\
\hline Onychoteuthis banksii & 2,03 & 1,41 & Lolliguncula (Loliolopsis) diomedeae & 1,71 & & 1,87 & \\
\hline Octopoteuthis spp. & 2,82 & 0,52 & Mastigoteuthis spp. & 12,08 & 0,82 & 1,93 & 8,00 \\
\hline Octopus spp. & 0,13 & 4,59 & Octopoteuthis spp. & 2,39 & & 0,52 & \\
\hline Sthenoteuthis oualaniensis & 0.48 & 3,14 & Octopus spp. & & 1,63 & 0,99 & 47,63 \\
\hline Sthenote unis oulatantensis & 0,40 & 3,14 & Ommastrephes bartramii & & & 0,02 & \\
\hline Otros moluscos & 2,12 & 0,92 & Pholidoteuthis boschmaii & 0,81 & & & \\
\hline Sub-total & 51,96 & 63,26 & Restos de cefalópodos & 8,85 & 0,41 & 0,10 & 2,83 \\
\hline & & & Sthenoteuthis oualaniensis & 4,37 & & 1,07 & 16,27 \\
\hline Osteichthyes & & & Thysanoteuthis rhombus & & & 0,02 & \\
\hline Auxis thazard & 1,09 & 2,14 & & & & & \\
\hline Anchoa spp. & 10,33 & 2,80 & Osteichthyes & & & & \\
\hline Merluccius gayi & 4,69 & 7,30 & Anchoa spp. & 2,28 & 2,45 & 2,30 & \\
\hline Otros peces & 31.85 & 2026 & Auxis thazard & 8,59 & & 0,62 & \\
\hline Utros peces & 31,85 & 20,20 & Benthosema panamense & 0,35 & & 0,28 & \\
\hline Sub-total & 47,96 & 32,5 & Chloroscombrus orqueta & 0,37 & & & \\
\hline & & & Hemanthis peruanus & & & 0,03 & \\
\hline Crustacea & & & Larimus argenteus & 0,38 & & 0,07 & \\
\hline Penaeus spp. & 0,07 & 4,51 & Larimus spp. & 0,35 & & & \\
\hline Sub-total & 0,07 & 4,51 & Merluccius gayi & 8,40 & & 7,48 & \\
\hline & & & Muraena spp. & 0,45 & & & \\
\hline & & & Ophichthus pacifici & 1,67 & & & \\
\hline & & & Ophichthus spp. & 0,39 & & 0,02 & \\
\hline & & & Oxyporhamphus micropterus & 0,41 & & & \\
\hline & & & Restos de Paralychthidae & & & 0,02 & \\
\hline & & & Restos de Scombridae & 0,35 & & & \\
\hline & & & Restos de Serranidae & & & & 2,06 \\
\hline & & & Restos de peces no identificados & 22,84 & 26,17 & 10,02 & 0,93 \\
\hline & & & Crustacea & & & & \\
\hline & & & Penaeus spp. & 0,39 & & 1,50 & \\
\hline & & & Restos de camarón & 0,38 & & 1,55 & \\
\hline
\end{tabular}




\section{Tabla 3}

Índice de traslapamiento trófico (Cë) y amplitud del nicho trófico (Bi) por estadio de madurez y sexo de $S$. lewini

Trophic overlapping index (Cë) and trophic niche width (Levin’s Bi) by maturity stage and sex of S. lewini

\begin{tabular}{lllccc}
\hline \multirow{2}{*}{$\begin{array}{c}\text { Estado de } \\
\text { madurez }\end{array}$} & Sexo & \multicolumn{2}{c}{ Juveniles } & \multicolumn{2}{c}{ Adultos } \\
\hline \multirow{2}{*}{ Juveniles } & & Machos & Hembras & Machos & Hembras \\
& Machos & 1 & & & \\
\multirow{2}{*}{ Adultos } & Hembras & 0,06 & 1 & & \\
& Machos & 0,23 & 0,32 & 1 & 1 \\
& Hembras & 0,12 & 0,16 & 0,23 & 0,22 \\
\hline
\end{tabular}

En los machos se identificaron 28 presas, de las cuales, 12 fueron cefalópodos, 13 peces y un crustáceo. De acuerdo con el \%IIR, las presas más importantes fueron: D. gigas (17,2\%), Histioteuthis spp. (16,4\%) y Anchoa spp. (10,3\%) (Tabla 2a). En las hembras se identificaron 27 presas, de las cuales 14 fueron cefalópodos, 10 peces y un crustáceo. En relación al \%IIR de las hembras, las presas más importantes fueron Histioteuthis spp. con 28,1\% y D. gigas con 18,3\% (Tabla 2a).

Con respecto a los índices ecológicos, se observó que el índice de amplitud de dieta $(\mathrm{Bi})$, mostró que tanto los machos $(B i=0,40)$ como hembras $(B i=0,43)$ de $S$. lewini, son organismos selectivos debido al mayor consumo de algunas presas, en particular de cefalópodos.

\section{Análisis trófico por estado de madurez sexual}

Entre adultos y juveniles se observó que pocas presas eran compartidas. De las 34 presas que conforman el espectro dietario de Sphyrna lewini, sólo cuatro fueron compartidos entre machos adultos y juveniles, mientras que siete fueron compartidas entre hembras adultas $\mathrm{y}$ juveniles (Tabla 2b).

En los juveniles de Sphyrna lewini, la presa de mayor importancia, según el \%IIR, en las hembras fue el cefalópodo D. gigas, el cual aportó 58,5\%; sin embargo este cefalópodo estuvo ausente en los machos (Tabla 2b). Las presas más importantes en los machos juveniles fue Mastigoteuthis spp. (12,1), Histioteuthis spp. (10,2) y A. lesueuri $(7,9)$ (Tabla 2b).

En los machos adultos de Sphyrna lewini, las especies más importantes fueron: D. gigas (53,7\%) e Histioteuthis spp. (14,9\%); mientras que las hembras adultas consumían principalmente Octopus spp. (47,6\%), Histioteuthis spp. (18,7\%) y S. oualaniensis (16,3\%) (Tabla 2b).
El índice de traslapamiento trófico $(\mathrm{C} \lambda)$, indicó que la dieta alimenticia para machos y hembras del tiburón martillo es muy similar ( $\mathrm{C} \lambda=0,81)$; sin embargo al separar la información por estadios de madurez y sexo, se observa que las dietas difieren, encontrando un traslapamiento más cercano entre hembras juveniles y machos adultos $(\mathrm{C} \lambda=0,32)$ (Tabla 3$)$, debido a un mayor consumo de Dosidicus gigas e Histioteuthis spp.

\section{Discusión}

El espectro trófico de Sphyrna lewini obtenido en el presente trabajo tiene similitud con los espectros tróficos registrados en otras partes del mundo, principalmente por la presencia de cefalópodos y peces como componente principal en su dieta. Klimley (1982), Galván et al. (1989), Aguilar-Castro (2003) y Torres-Rojas et al. (2006) encontraron que $S$. lewini en el Golfo de California se alimenta de cefalópodos, peces y crustáceos, al igual que lo presentado en este estudio, donde los cefalópodos ( $D$. gigas e Histioteuthis spp.) constituyeron las presas principales, mientras que los peces y crustáceos tuvieron una menor representación. Smale \& Cliff (1998) determinaron que $S$. lewini en Sudáfrica, consume principalmente cefalópodos Octopodidae, Octopoteuthidae y Ancistrocheiridae, familias que también fueron encontradas en el presente estudio, donde las de mayor importancia fueron Histioteuthidae y Ommastrephidae.

En un estudio de dieta en Sphyrna lewini realizado en aguas ecuatorianas por Castañeda \& Sandoval (2007), se registraron como presas principales a los cefalópodos Dosidicus gigas, Mastigoteuthis dentata y Ancistrocheirus lesueuri; lo que coincide parcialmente con el presente estudio ya que $D$. gigas fue una de las presas principales, junto con Histioteuthis spp. 
Existen pocas investigaciones donde se registra la presencia de adultos y juveniles de Sphyrna lewini en la misma zona, como sucede en Ecuador, ya que en otras regiones del mundo es común encontrar solo juveniles de esta especie, como sucede en el Golfo de California (costa suroccidental), en la cual el alimento de machos juveniles se basa en peces epipelágicos como Scomber japonicus y Sardinops caeruleus, así como del cefalópodo mesopelágico Abraliopsis affinis; mientras que las hembras juveniles se alimentan de los cefalópodos Dosidicus gigas y Onychoteuthis banksii, indicando una segregación por sexos debido al hábitat trófico, donde los machos juveniles se alimentaron más en zonas costeras y de la region epipelágica; mientras que las hembras juveniles consumen su alimento en aguas oceánicas (Aguilar-Castro 2003). En esta misma zona del Golfo de California (costa suroriental), Torres-Rojas et al. (2006) registraron que los juveniles de S. lewini consumen principalmente al cefalópodo costero Loliolopsis diomedeae, así como los peces costeros de las familias Carangidae y Gerreidae. En la presente investigación en Ecuador, juveniles de S. lewini se alimentaron principalmente de cefalópodos (D. gigas, Octopus spp., Histioteuthis spp., S. oualaniensis y Mastigoteuthis spp.); mientras que Castañeda \& Sandoval (2007) para esta misma zona de Ecuador, registraron que los juveniles se alimentaron principalmente de $D$. gigas y L. (Loliolopsis) diomedeae. Los diferentes estudios realizados en diferentes partes del mundo indican que los juveniles de tiburón martillo pueden alimentarse tanto de presas costeras, como de presas oceánicas con mayor preferencia de cefalópodos.

Con respecto a los adultos de Sphyrna lewini, en el Golfo de California consumieron principalmente cefalópodos en la zona oceánica (Galván et al. 1989). En aguas ecuatorianas, Castañeda \& Sandoval (2007) registran a los cefalópodos A. lesueuri, D. gigas y Octopoteuthis sicula, como las presas más importantes en adultos. En el presente estudio las hembras adultas consumieron principalmente Octopus spp., Sthenoteuthis ovalaniensis e Histioteuthis spp., a diferencia de los machos adultos, los cuales consumieron en mayor proporción D. gigas e Histioteuthis spp.

La ausencia de D. gigas en las hembras adultas y la mayor proporción de Octopus spp. en sus estómagos, indican que existe una segregación por sexo en los tiburones adultos, donde las hembras se alimentan de presas más cercanas al bentos, al consumir Octopus spp.; mientras que los machos adultos permanecen en la zona oceánica consumiendo $D$. gigas, el cual es un calamar que realiza migraciones verticales y permanece a profundidades de 350 a 400 m durante el día, migrando a profundidades de 100 a $200 \mathrm{~m}$ durante la noche (Gilly et al. 2006) y es probable que los machos adultos los consuman durante estas migraciones nocturnas de los calamares.

De acuerdo a los resultados obtenidos en este estudio, se considera que $S$. lewini es un depredador especialista $(B i=0,35)$ ya que muestra preferencia por los cefalópodos (Histioteuthis spp. y D. gigas). Esta misma conclusión se obtuvo en los trabajos de Aguilar-Castro (2003) y Castañeda \& Sandoval (2007); sin embargo, Torres-Rojas et al. (2006) consideran a S. lewini como un depredador generalista, debido que estos autores consideraron solo tiburones juveniles, del sureste del Golfo de California, los cuales depredaban sobre un mayor número de presas de origen costero.

Los tiburones martillo juveniles, usualmente menores de $180 \mathrm{~cm}$, son de hábitos tróficos costeros, conforme van creciendo y madurando sexualmente, necesitan más requerimientos energéticos, por lo cual consumen presas que les aporten mayor biomasa como son los cefalópodos de la zona oceánica; sin embargo, fue interesante observar que los machos adultos y las hembras juveniles presentaron presas comunes como son D. gigas e Histioteuthis spp., lo cual podría indicar que su hábitat trófico es más oceánico. Asimismo los juveniles de este tiburón además de consumir cefalópodos de zonas oceánicas, se alimentan en menor proporción de presas de hábitat costero y bentónico como son la merluza Merluccius gayi, Lolliguncula (Loliolopsis) diomedeae, Muraena spp., Ophichthus spp., Synodus spp., Larimus argenteus y Penaeus spp.

Este estudio ha mostrado el gran espectro trófico del tiburón martillo, Sphyrna lewini, lo que coincide con lo reportado por otros estudios. Usualmente, estos tiburones son más bien especialistas dado a que en general consumen cefalópodos, independiente de su localidad. Las diferencias dietarias estarían determinadas principalmente por el estado de madurez, lo cual indicaría una ocupación diferencial de hábitat debido a los requerimientos energéticos de juveniles y adultos que inducen a la captura de distintos tipos de presas.

\section{Agradecimientos}

A David Castañeda, Alejandro Sandoval, Carlos Polo Silva, Álvaro Baigorrí, Fabián Pacheco, José Méndez, Vanesa Velásquez, Liliana Rendón, a los comerciantes de Playa Tarqui (Manabi-Ecuador). A Leonardo CastilloGéniz, Juan Carlos Pérez-Jiménez y los revisores anónimos por la revisión del manuscrito. Al IPN (COFAA y EDI) por el apoyo otorgado a F. Galván. 


\section{Literatura citada}

Aguilar-Castro N. 2003. Ecología trófica de juveniles del tiburón martillo Sphyrna lewini (Griffith \& Smith, 1834) en el Golfo de California. Tesis de Maestría CICIMARIPN, La Paz, 121 pp.

Bejarano AM. 2007. Biología reproductiva del tiburón martillo Sphyrna lewini (Griffith y Smith, 1834) en Salina Cruz, Oaxaca, México. Tesis de Maestría. CICIMAR-IPN, La Paz, 74 pp.

Castañeda SD \& LA Sandoval. 2007. Hábitos alimentarios del tiburón martillo Sphyrna lewini (Griffith \& Smith, 1834) en el Pacifico ecuatoriano. En: Martínez-Ortíz J \& F GalvánMagaña (eds). Tiburones en el Ecuador: Casos de estudio / Sharks in Ecuador: Case studies, pp. 66-76. EPESPO PMRC, Manta.

Chirichigno N. 1998. Clave para identificar los peces marinos del Perú, 496 pp. Publicación Especial del Instituto del Mar del Perú, Callao.

Clarke MR. 1986. A handbook for the identification of cephalopod beaks, 273 pp. Clarendon Press, Oxford.

Clothier CR. 1950. A key to some southern California fishes based on vertebral characters. California Department of Fish and Game, Fish Bulletin 79: 1-83.

Colwell RK. 2000. EstimateS: Statistical estimation of species richness and shared species from samples (Software and User's Guide), Version 6.0. University of Connecticut, USA. [CD-ROM]

Compagno LJV. 1984. Sharks of the world. An annotated and illustrated catalogue of sharks species known date. Part 12. Hexanchiformes to Carcharhiniformes. FAO Fisheries Synopsis 125(4): 1-655.

Cortés E. 1997. A critical review of methods of studying fish feeding base on analysis of stomach contents: application to elasmobranch fishes. Canadian Journal of Fisheries and Aquatic Sciences 54: 726-738.

Fischer W, F Krupp, W Schneider, C Sommer, KE Carpenter \& VH Niem. 1995. Guía FAO para la identificación de especies para los fines de pesca. Pacífico Centro-Oriental, 1813 pp. FAO, Roma.

Galván MF, H Nienhuis \& P Klimley. 1989. Seasonal abundance and feeding habits of sharks of the lower Gulf of California Mexico. California Fish and Game 75(2): 74-84.

García GI. 2001. Patrones morfológicos del otolito Sagitta de algunos peces óseos del mar peruano. Boletín del Instituto del Mar del Perú 20 (1/2): 4-83.

Gilly WF, U Markaida, CH Baxter, BA Block, A Boustany, L Zeidberg, K Reisenbichler, B Robison, G Bazzino \& C Salinas. 2006. Vertical and horizontal migrations by the jumbo squid Dosidicus gigas revealed by electronic tagging. Marine Ecology Progress Series 324: 1-17.
Hyslop E. 1980. Stomach contents analysis: a review of methods and their application. Journal of Fish Biology 17: 411-429.

Klimley AP. 1981. Grouping behaviour in the scalloped hammerhead. Oceanus 24(4): 65-71.

Klimley AP. 1982. Social organization of schools of the scalloped hammerhead, Sphyrna lewini (Griffith and Smith), in the Gulf of California. Ph.D. Dissertation, University of California, San Diego, La Jolla, 341 pp.

Klimley AP \& DR Nelson. 1981. Schooling of the scalloped hammerhead shark, Sphyrna lewini, in the Gulf of California. Fishery Bulletin 79(2): 356-360.

Krebs C. 1989. Ecological methodology, 550 pp. Harper and Row, New York.

Labropoulou M \& A Eleftheriou. 1997. The foraging ecology of two pairs of congeneric demersal fish species: importance of morphological characteristics in prey selection. Journal of Fish Biology 50: 324-340.

Langton R. 1982. Diet overlap between the Atlantic cod Gadus morhua, silver hake Merluccius bilinearis and fifteen other Northwest Atlantic finfish. Fishery Bulletin 80: 745-759.

Pinkas L, SM Oliphant \& ILK Iverson. 1971. Food habits of albacore, bluefin tuna, and bonito in California waters. Department Fish and Game. Fish Bulletin 152: 1-105.

Robertson DR \& G Allen. 2002. Peces costeros del Pacífico Oriental Tropical: Un sistema de información. Instituto Smithsonian de Investigaciones Tropicales. Balboa Panamá. [CD-ROM].

Rubio RE. 1988. Peces de importancia comercial para el Pacífico Colombiano, 483 pp. Departamento de Biología, Universidad del Valle, Cali.

Smale MJ \& G Cliff. 1998. Cephalopods in the diets of four shark species (Galeocerdo cuvier, Sphyrna lewini, Sphyrna zygaena y S. mokarran) from Kwazulu-Natal, South Africa. South African Journal of Marine Science 20: 241-253.

Smith P \& M Zaret. 1982. Bias in estimating niche overlap. Ecology 63(5): 1248-1253.

StatSoft 2001. STATISTICA. Date analysis software system and computer program manual. Versión 6. StatSoft, Inc., Tulsa.[CD-ROM].

Torres-Rojas YE, A Hernández \& F Galván. 2006. Feeding habits of the scalloped hammerhead Sphyrna lewini, in Mazatlán waters, southern Gulf of California, Mexico. Cybium 30(4): 85-90.

Wolff CA. 1984. Identification and estimation of size from the beaks of eighteen species of cephalopods from the Pacific Ocean. NOAA Technical Report, NMFS 17: 1-50. 